13. Kestila, M., et al. 1998. Positionally cloned gene for a novel glomerular protein - nephrin - is mutated in congenital nephrotic syndrome. Mol. Cell. 1:575-582.

14. Schwartz, E.J., et al. 2001. Human immunodeficiency virus- 1 induces loss of contact inhibition in podocytes. J. Am. Soc. Nephrol. 12:1677-1684.

15. Barisoni, L., Kriz, W., Mundel, P., and D'Agati, V. 1999. The dysregulated podocyte phenotype: a novel concept in the pathogenesis of collapsing idiopathic focal segmental glomerulosclerosis and HIV-associated nephropathy. J. Am. Soc. Nephrol. 10:51-61.

16. Korgaonkar, S.N., et al. 2008. HIV-1 upregulates VEGF in podocytes. J. Am. Soc. Nephrol. 19:877-883.

17. Winston, J.A., et al. 2001. Nephropathy and establishment of a renal reservoir of HIV type 1 during primary infection. N. Engl. J. Med. 344:1979-1984.

18. Eggers, P.W., and Kimmel, P.L. 2004. Is there an epidemic of HIV Infection in the US ESRD program? J. Am. Soc. Nephrol. 15:2477-2485.

19. Kopp, J.B., et al. 2008. MYH9 is a major-effect risk gene for focal segmental glomerulosclerosis. Nat. Genet. 40:1175-1184.

20. Kao, W.H., et al. 2008. MYH9 is associated with nondiabetic end-stage renal disease in African Americans. Nat. Genet. 40:1185-1192.

21. Heath, K.E., et al. 2001. Nonmuscle myosin heavy chain IIA mutations define a spectrum of autosomal dominant macrothrombocytopenias: MayHegglin anomaly and Fechtner, Sebastian, Epstein, and Alport-like syndromes. Am. J. Hum. Genet. 69:1033-1045.

22. Kelley, M.J., Jawien, W., Ortel, T.L., and Korczak, J.F.
2000. Mutation of MYH9, encoding non-muscle myosin heavy chain A, in May-Hegglin anomaly. Nat. Genet. 26:106-108.

23. Seri, M., et al. 2000. Mutations in MYH9 result in the May-Hegglin anomaly, and Fechtner and Sebastian syndromes. The May-Heggllin/Fechtner Syndrome Consortium. Nat. Genet. 26:103-105.

24. Papeta, N., et al. 2009. Susceptibility loci for murine HIV-associated nephropathy encode transregulators of podocyte gene expression. J. Clin. Invest. 119:1178-1188.

25. Gharavi, A.G., et al. 2004. Mapping a locus for susceptibility to HIV-1-associated nephropathy to mouse chromosome 3. Proc. Natl. Acad. Sci. U. S. A. 101:2488-2493.

26. Chen, Y., et al. 2008. Variations in DNA elucidate molecular networks that cause disease. Nature. 452:429-435.

27. Schadt, E.E., et al. 2005. An integrative genomics approach to infer causal associations between gene expression and disease. Nat. Genet. 37:710-717.

28. Pravenec, M., et al. 2008. Identification of renal $\mathrm{Cd} 36$ as a determinant of blood pressure and risk for hypertension. Nat. Genet. 40:952-954.

29. Tsukaguchi, H., et al. 2002. NPHS2 mutations in late-onset focal segmental glomerulosclerosis: R229Q is a common disease-associated allele. J. Clin. Invest. 110:1659-1666.

30. Huber, T.B., et al. 2006. Podocin and MEC-2 bind cholesterol to regulate the activity of associated ion channels. Proc. Natl. Acad. Sci. U. S. A. 103:17079-17086.

31. Huber, T.B., et al. 2003. Molecular basis of the functionalpodocin-nephrin complex: mutations in the
NPHS2 gene disrupt nephrin targeting to lipid raft microdomains. Hum. Mol. Genet. 12:3397-3405.

32. Hubner, N., et al. 2005. Integrated transcriptional profiling and linkage analysis for identification of genes underlying disease. Nat. Genet. 37:243-253.

33. Ratelade, J., et al. 2008. Maternal environment interacts with modifier genes to influence progression of nephrotic syndrome. J. Am. Soc. Nephrol. 19:1491-1499.

34. Quaggin, S.E. 2002. Transcriptional regulation of podocyte specification and differentiation. Microsc. Res. Tech. 57:208-211.

35. Brem, R.B., Yvert, G., Clinton, R., and Kruglyak, L. 2002. Genetic dissection of transcriptional regulation in budding yeast. Science. 296:752-755.

36. Somlo, S., and Mundel, P. 2000. Getting a foothold in nephrotic syndrome. Nat. Genet. 24:333-335.

37. Kopp, J.B., et al. 1992. Progressive glomerulosclerosis and enhanced renal accumulation of basement membrane components in mice transgenic for human immunodeficiency virus type 1 genes. Proc. Natl. Acad. Sci. U. S. A. 89:1577-1581.

38. Zuo, Y., et al. 2006. HIV-1 genes vpr and nef synergistically damage podocytes, leading to glomerulosclerosis. J. Am. Soc. Nephrol. 17:2832-2843.

39. Zhong, J., et al. 2005. Expression of HIV-1 genes in podocytes alone can lead to the full spectrum of HIV-1associated nephropathy. Kidney Int. 68:1048-1060.

40. Husain, M., et al. 2005. HIV-1 Nef induces dedifferentiation of podocytes in vivo: a characteristic feature of HIVAN. AIDS. 19:1975-1980.

41. Chan, K.T., et al. 2009. Accelerated development of collapsing glomerulopathy in mice congenic for the HIVAN1 locus. Kidney Int. 75:366-372.

\title{
Dietary sugars: a fat difference
}

\author{
Susanna M. Hofmann' ${ }^{1}$ and Matthias H. Tschöp1,2,3
}

1Department of Medicine and 2Department of Psychiatry, Obesity Research Center, University of Cincinnati College of Medicine, Cincinnati, Ohio, USA.
${ }^{3}$ Department of Pharmacology, German Institute of Human Nutrition Potsdam-Rehbrücke, Nuthetal, Germany.

\begin{abstract}
Coronary heart disease is a major cause of morbidity and mortality in Western societies. The metabolic syndrome, characterized by obesity, insulin resistance, elevated blood pressure, elevated triglycerides, and low levels of high-density lipoprotein cholesterol, confers substantial risk of coronary heart disease. Current pathogenetic models suggest that postprandial hyperlipidemia is one specific metabolic abnormality that is typically associated with increased morbidity. In this issue of the JCI, Stanhope and colleagues demonstrate that consumption of fructose-sweetened but not glucose-sweetened beverages for 10 weeks increases de novo lipid synthesis, promotes dyslipidemia, impairs insulin sensitivity, and increases visceral adiposity in overweight or obese adults (see the related article beginning on page 1322).
\end{abstract}

There is widespread agreement that a chronic, dietary-induced increase of adipos-

Conflict of interest: M.H. Tschöp is a member of the Marcadia Biotech Scientific Advisory Board. Research in the Tschöp laboratory is funded by Marcadia Biotech, Ambrx Inc., and Ethicon Endo-Surgery Inc.

Nonstandard abbreviations used: CVD, cardiovascular disease; HFCS, high-fructose corn syrup; LPL, lipoprotein lipase; PGC-1 $\beta$, PPAR $\gamma$ coactivator-1 $\beta$; sdLDL, small dense LDL.

Citation for this article: J. Clin. Invest. 119:1089-1092 (2009). doi:10.1172/JCI39332. ity in humans, beyond a BMI of $30 \mathrm{~kg} / \mathrm{m}^{2}$, is an unhealthy condition. In the event that any readers harbor some remaining skepticism, an unprecedented thorough analysis in close to 900,000 participants from almost 60 prospective studies was very recently published, proving beyond any possible doubt that progressive excess mortality is caused by increased body adiposity: Survival was found to be reduced by $2-10$ years as a consequence of a BMI in the range of $30-40 \mathrm{~kg} / \mathrm{m}^{2}(1)$.

\section{Dietary sugars and obesity}

The average American consumed $64 \mathrm{~kg}$ of added (!) sugars and sweeteners in 2005, a 19\% increase since 1970 (2). Increased use of high-fructose corn syrup (HFCS) as a sweetener (as frequently used in many sweetened beverages, including carbonated sodas) over the last several decades has been proposed as one potentially important dietary factor that may have contributed to the widespread and life-shortening increase in human obesity observed in Westernized societies. While "normal sugar," also known as sucrose, contains equimolar amounts of fructose and glucose, HFCS contains about $5 \%$ more fructose than glucose. HFCS is manufactured by hydrolyzing corn starch into glucose, which then is partly isomerized into fructose by enzymatic measures. Fructose is preferred by food and soft drink manufacturers due to the fact that fructose exerts a significantly increased perception of sweetness. When we (the authors) were first exposed to clinical medicine, we were still told that small 
amounts of fructose could be recommended as a dietary sugar that offered metabolic benefits in patients with diabetes. However, public opinion later changed to the opposing view, and the use of dietary fructose as a sweetener was accused of having contributed to if not caused the ongoing obesity "epidemic.” Exaggerated fearmongering regarding fructose intake sometimes went overboard, to the extent that consumption of fresh fruits was questioned as potentially metabolically unfavorable due to the associated ingestion of fructose. Currently, scientific opinion has become more tempered between these two extremes, with widespread agreement that chronic consumption of large amounts of dietary fructose may have adverse metabolic effects. It is also clear that fresh fruit remains a highly recommended dietary component, as one would have to eat vast quantities of fruits every day in order to ingest metabolically adverse amounts of dietary fructose. However, a complete scientific understanding of why human metabolic processes may react differentially to concentrated fructose as compared to other dietary sugars, such as glucose, remained largely unknown. While a number of interesting studies, conducted primarily in rodent models, indicating a number of potentially relevant differences between the metabolism of fructose and glucose were published in the recent past $(3,4)$, Stanhope and colleagues have now taken our knowledge of the metabolism of these sugars in humans to the next level with a carefully designed study published in this issue of the JCI, in which they have comprehensively compared the metabolic impact of these two major dietary sugars in human subjects (5).

\section{Not all sugars are equal in lipid metabolism}

In this study, Stanhope et al. (5) set out to determine whether the consumption of fructose- or glucose-sweetened beverages by humans at $25 \%$ of their energy requirements for 10 weeks would result in body weight gain, have adverse effects on lipid metabolism, and decrease insulin sensitivity and whether such an impact would differ between men and women. State-of-the-art metabolic measurements were applied with great attention to detail, thereby providing the most informative clinical study available to date to address these questions: 32 male and female subjects (average age: 50 years; BMI: 29) consumed either fructose$(n=17)$ or glucose-sweetened $(n=15)$ beverages at $25 \%$ of energy requirements. The study design consisted of a 2-week baseline period during which subjects resided in a clinical research center and consumed an energy-balanced diet high in complex carbohydrates and no fructose- or glucosesweetened beverages; an 8-week outpatient intervention period during which the subjects consumed fructose- or glucose-sweetened beverages as part of a self-selected ad libitum diet; and finally, a second 2 -week inpatient period during which the fructoseor glucose-sweetened beverages were consumed as part of an energy-balanced diet.

The study by Stanhope and colleagues (5) does not address all of the questions with respect to the metabolic effects of dietary sugars, and the outcome does not justify any simple dietary recommendation. However, a number of novel and clinically relevant scientific conclusions can be solidly drawn based on their results. Importantly, individuals in both groups (consuming fructose- or glucose-sweetened beverages) exhibited comparable body weight gain throughout the study. In contrast to expectations, fasting triglyceride levels were actually somewhat increased in subjects consuming glucose-sweetened beverages, while these levels were unchanged in subjects consuming fructose-sweetened beverages. However, that observation was one of a few exceptions. Most other observations indicated that consumption of dietary fructose had unfavorable effects on lipid metabolism: consumption of fructose-sweetened but not glucose-sweetened beverages for 10 weeks increased the synthesis of lipids in the liver (de novo lipogenesis) as well as the total volume of intraabdominal fat depots (known to be have more adverse health effects than subcutaneous fat depots) in the overweight adult participants. In subjects consuming glucose-sweetened beverages, only the volume of the subcutaneous fat depots (believed to be less detrimental to metabolic health) increased. Consumption of fructose-sweetened but not glucose-sweetened beverages also led to larger increases of postprandial and 24-hour plasma triglyceride levels. Fructose-induced alterations of several processes, including hepatic lipid synthesis and reduced lipid clearance, appear to have contributed to that outcome, as Stanhope and colleagues show, using tracer studies and measurements of lipoprotein lipase (LPL) activity. The apparently largely fructose-specific nutrient-induced changes in lipid metabolism were not limited to postprandial triglyceride concentrations, storage efficiency, and regional distribution of triglycerides; levels of apoB, LDL cholesterol, small, dense LDL (sdLDL), oxidized LDL-cholesterol, remnant lipoprotein triglyceride, and the apoB/apoA1 ratio (all biomarkers of increased risk for cardiovascular disease [CVD]) were also increased during consumption of fructose and unchanged during consumption of glucose. One intriguing facet in these findings was a pattern throughout the chronic dietary treatment study in which postprandial fat metabolism showed greater differences between the two sugar-consuming groups of volunteers than under fasting conditions.

\section{Differential effects on glucose tolerance}

While the predominant differences between those individuals consuming fructosesweetened beverages and those consuming glucose-sweetened beverages were most prominently associated with alterations of lipid metabolism, Stanhope and colleagues also observed significant differences in glucose tolerance and insulin sensitivity between the two groups of subjects: fasting glucose concentrations decreased in subjects consuming glucose-sweetened beverages but increased in subjects consuming fructose-sweetened beverages (5). Similarly, fasting insulin concentrations as well as glucose and insulin excursions during an oral glucose tolerance test were increased during consumption of fructose-sweetened beverages only, and the insulin-sensitivity index, as assessed by deuterated glucose disposal, was decreased exclusively in subjects consuming fructose-sweetened beverages. The authors propose that this impact on glucose metabolism may be a consequence of increases of hepatic diacylglycerol levels in the subjects consuming fructose-sweetened beverages. Finally, the consumption of dietary fructose exhibited differential effects in that it increased visceral fat mass more in men and decreased insulin sensitivity more in women, suggesting clear sexual phenotype differences in the specific metabolic effects of these 2 major dietary sugars.

In summary, Stanhope and colleagues provide major scientific progress by demonstrating marked differences in the metabolic effects of these two major sugars with respect to their ability to promote intraabdominal lipid deposition and hepatic lipid production, while shifting cholesterol metabolism in an unfavorable manner and diminishing insulin sensitivity in humans (5). These marked differences were observed despite comparable mean 
weight gain in the two groups of subjects consuming glucose- or fructose-sweetened beverages. However, the study stops short of showing whether fructose consumption would decrease insulin sensitivity to the same degree in the absence of visceral fat gain. Statistical analysis of the available descriptive data appears to suggest that the changes of body fat and insulin sensitivity are not necessarily codependent.

\section{Fructose: a sugar with a history}

These findings (5) significantly extend a long-standing history of scientific evidence on the differential metabolic effects of dietary sugars, as previously reviewed (6-9). In particular, and unlike glucose, fructose is transported into the cell in an insulin-independent, GLUT-5-mediated manner and is converted into trioses that are essential backbones for the de novo synthesis of triglycerides and cholesterol. Hence, fructose promotes biosynthesis and accumulation of these lipids more efficiently than glucose does. Accordingly, fructose was shown to exert specific and distinguishable effects on lipid metabolism more than four decades ago (10). Moreover, fructose was shown to acutely deplete ATP in the liver due to its rapid initial phosphorylation to fructose1-phosphate (11). This fructose-induced hepatic energy deprivation has been proposed to be a cause of fructose-related, nonalcoholic fatty liver disease (NAFLD) (12), which has been shown to be an important factor in the pathogenesis of insulin resistance and type 2 diabetes (13). Likewise, the increased availability of fructose-1-phosphate may also lead to increased uric acid biosynthesis. Accordingly, fructose consumption has been linked to increased incidence of gout (14). Notably, uric acid has been shown to contribute to atherosclerosis (15), suggesting that fructose promotes the formation of several risk factors for CVD, including uric acid, triglycerides, and cholesterol, and according to the present study by Stanhope and colleagues (5), specific changes in apolipoprotein metabolism.

\section{Do fructose-induced changes in lipid metabolism confer increased cardiovascular risk?}

The present study (5) adds an important missing piece to the very complex puzzle of diet-induced influences on the pathogenesis of CVD. However, in light of the large body of data available on links between lipid metabolism and cardiovascular risk, the data presented here fall short of providing a complete understanding of the exact role dietary fructose may play within these extremely complicated and multilayered pathophysiological interplays. The current accepted model of pathogenesis suggests that postprandial hyperlipidemia is a characteristic metabolic abnormality of a number of lifestyle-related conditions that are associated with increased morbidity (such as hypertriglyceridemia, metabolic syndrome, obesity, and type 2 diabetes) and mortality from CVD. The perception that carbohydrate intake and not fat intake has a positive association with CVD has put the spotlight on postprandial lipid metabolism. Fructose, as a preferred substrate for lipogenesis, is known from rodent studies to enhance VLDL accumulation in and secretion from the liver, which is consistent with increased hepatic triglyceride synthesis. Although the authors (5) show nicely that postprandial hepatic de novo lipogenesis in humans is markedly enhanced during chronic exposure to fructose, it is unclear whether this process can explain the observed marked postprandial elevation of triglycerides and remnant-like particle lipoproteins. Recent findings (16) indicate that chronic fructose feeding in hamsters leads to intestinal apoB48 overproduction and de novo lipogenesis, suggesting that organs other than the liver may be involved, but this has not been studied here by Stanhope and colleagues. The reduced postprandial LPL activity in subjects consuming fructose suggests that a reduced capacity to hydrolyze chylomicrons and VLDLs could also contribute to the observed postprandial hypertriglyceridemia. It would therefore be of interest to determine whether fructose may directly inhibit LPL activity, although the likelihood of dietary fructose reaching LPL in peripheral tissues may be limited. In addition, the possibility that fructose acutely alters liver capacity to clear chylomicron remnants cannot be excluded, and the observation by Stanhope and colleagues that sdLDL concentration is increased by dietary fructose intake may reflect impaired LDL receptor-mediated uptake. As a consequence, sdLDL half-life would be prolonged, leading to increased oxidative modification and transport into the subendothelial space, which in turn would augment the atherogenic potential, thereby offering one potential mechanistic link between chronic fructose overconsumption and CVD. A finding that stands out as counterintuitive in the present study is the higher fasting HDL-cholesterol level in the subjects consuming fructose-sweetened beverages as compared with the glucose-sweetened beverage group. Why does the experimental group with the more atherogenic sdLDL particles also exhibit an increase in HDL-cholesterol, which is widely thought to be protective against the development of atherosclerosis (17)? This observation is yet another indicator that metabolic differences between fructose and glucose metabolism are not clear cut, underlining how important it is to keep in mind that the actual long-term impact of fructose overconsumption compared with glucose overconsumption on atherosclerosis and CVD remains unknown.

What also remain unknown are the specific molecular mechanisms beyond subtle biochemical differences that explain differential metabolic effects of fructose and glucose. Stanhope et al. (5) rightfully point out that in contrast to glucose, the metabolism of fructose is largely independent of hepatic phosphofructokinase regulation; thus its uptake by the liver and its metabolism to de novo lipid substrate is not limited by cytosolic ATP and citrate availability. However, a recent report by Shulman and colleagues suggests that an additional molecular mechanism plays a key role in fructose-induced lipid metabolism changes (18). These authors evaluated the role of PPAR $\gamma$ coactivator- $1 \beta$ (PGC-1 $\beta$ ), an important transcriptional coactivator modulating hepatic lipogenesis, in the pathogenesis of fructose-induced insulin resistance. Their results support a relevant role for PGC-1 $\beta$ in the pathogenesis of fructoseinduced de novo lipogenesis and insulin resistance. It seems theoretically possible that the fact that fructose is bypassing phosphofructokinase explains how dietary fructose promotes hepatic de novo lipogenesis, whereas fructose-induced modulation of PGC- $1 \beta$ activity may enhance the general lipogenic potential of the liver.

\section{Dietary sugars: a not so sweet future?}

Independent of the exact mechanism by which fructose differentially affects glucose and lipid metabolism in comparison with glucose, does the study reported by Stanhope and colleagues (5) teach us that we should avoid food items or beverages that contain significant amounts of fructose? Yes and no. The answer requires consideration of the forms in which dietary sugars 
are typically consumed. Rather than containing pure fructose or pure glucose, sweeteners typically used by the food industry are sucrose (50\% glucose and $50 \%$ fructose) or HFCS. It is not known whether the adverse effects of sucrose and HFCS consumption are "diluted" by their lower fructose content relative to pure fructose. In the absence of studies comparing the long-term effects of consuming HFCS and/or sucrose with $100 \%$ fructose, it is not certain what levels of these sweeteners might increase the risk for atherosclerosis, CVD, and type 2 diabetes. Dose-response studies of the metabolic effects of both fructose and HFCS/sucrose in different populations, particularly those at risk for metabolic disease, are needed to more fully address this question. However, what is now clear is that chronic overconsumption of dietary sugars in general is detrimental to our health and these effects may be synergistic with chronic increases in caloric intake. Nevertheless, a recent article in the New York Times (19) reports that even in the absence of long-term studies, public perception may already be anticipating results, since beverages promoted as not containing HFCS have started to surface in advertisements and the source of sugars or carbohydrates in general is becoming, more and more, a standard label on food items. Will restaurants in the near future be required to print not only the number of calories but also the type and source of the sugars contained in the foods next to each item on their menu? One of the most intriguing results of the study by Stanhope and colleagues actually indicates that body weight was stable during the inpatient period at the end of the study in which sugar-sweetened beverage consumption was continued but food intake was absolutely controlled such that the subjects were in neutral energy balance. However, during the 8-week outpatient intervention period, when the subjects consumed ad libitum, self-selected diets, both groups of subjects exhibited significant increases of body weight, fat mass, and waist circumference. Is a generally hypercaloric environment required for dietary sugars to have metabolically adverse effects? For our part, we will continue to aim for moderation of balanced caloric ingestion without excluding the occasional sweet soda. By the way, is anybody studying the potentially life-shortening psychological effects of having to go through chronic fructose-sweetened beverage withdrawal?

\section{Acknowledgments}

We thank Michael Ristow (Institute of Nutrition, University of Jena, Jena, Germany) for helpful scientific discussions.

Address correspondence to: Matthias H. Tschöp, Departments of Medicine and Psychiatry, Obesity Research Center, University of Cincinnati-College of Medicine, 2170 Galbraith Road, Cincinnati, Ohio 45237, USA. Phone: (513) 558-8648; Fax: (513) 5588658; E-mail: tschoemh@ucmail.uc.edu.

\footnotetext{
1. Prospective Studies Collaboration Group. 2009. Body-mass index and cause-specific mortality in 900,000 adults: collaborative analyses of 57 prospective studies. Lancet. Online publication ahead of print. doi:10.1016/S0140-6736(09)60318-4.

2. Wells, F.W., and Busby, J.C. 2008. Dietary assessment of major trends in US food consumption, 1970-2005. Economic Information Bulletin. 33:18.

3. Suga, A., et al. 2000. Effects of fructose and glucose on plasma leptin, insulin, and insulin resistance in lean and VMH-lesioned obese rats. Am. J. Physiol. Endocrinol. Metab. 278:E677-E683.

4. Cha, S.H., Wolfgang, M., Tokutake, Y., Chohnan, S., and Lane, M.D. 2008. Differential effects of central fructose and glucose on hypothalamic malonylCoA and food intake. Proc. Natl. Acad. Sci. U. S. A. 105:16871-16875.
}

5. Stanhope, K.L., et al. 2009. Consuming fructosesweetened, not glucose-sweetened, beverages increases visceral adiposity and lipids and decreases insulin sensitivity in overweight/obese humans. J. Clin. Invest. 119:1322-1334.

6. Wolf, A., Bray, G.A., and Popkin, B.M. 2008. A short history of beverages and how our body treats them. Obes. Rev. 9:151-164.

7. White, J.S. 2008. Straight talk about high-fructose corn syrup: what it is and what it ain't. Am. J. Clin. Nutr. 88:1716S-1721S.

8. Livesey, G., and Taylor, R. 2008. Fructose consumption and consequences for glycation, plasma triacylglycerol, and body weight: meta-analyses and meta-regression models of intervention studies. Am. J. Clin. Nutr. 88:1419-1437.

9. Brown, C.M., Dulloo, A.G., and Montani, J.P. 2008. Sugary drinks in the pathogenesis of obesity and cardiovascular diseases. Int. J. Obes. (Lond.). 32(Suppl. 6):S28-S34.

10. Kuo, P.T., and Bassett, D.R. 1965. Dietary sugar in the production of hypertriglyceridemia. Ann. Intern. Med. 62:1199-1212.

11. Mäenpää, P.H., Raivio, K.O., and Kekomaki, M.P. 1968. Liver adenine nucleotides: fructose-induced depletion and its effect on protein synthesis. Science. 161:1253-1254.

12. Cortez-Pinto, H., et al. 1999. Alterations in liver ATP homeostasis in human nonalcoholic steatohepatitis: a pilot study. JAMA. 282:1659-1664.

13. Tilg, H., and Moschen, A.R. 2008. Insulin resistance, inflammation, and non-alcoholic fatty liver disease. Trends Endocrinol. Metab. 19:371-379.

14. Choi, H.K., and Curhan, G. 2008. Soft drinks, fructose consumption, and the risk of gout in men: prospective cohort study. BMJ. 336:309-312.

15. Kanellis, J., and Kang, D.H. 2005. Uric acid as a mediator of endothelial dysfunction, inflammation, and vascular disease. Semin. Nephrol. 25:39-42.

16. Haidari, M., et al. 2002. Fasting and postprandial overproduction of intestinally derived lipoproteins in an animal model of insulin resistance. Evidence that chronic fructose feeding in the hamster is accompanied by enhanced intestinal de novo lipogenesis and ApoB48-containing lipoprotein overproduction. J. Biol. Chem. 277:31646-31655.

17. Rader, D.J. 2007. Illuminating HDL - is it still a viable therapeutic target? N. Engl.J. Med. 357:2180-2183.

18. Nagai, Y., et al. 2009. The role of peroxisome proliferator-activated receptor gamma coactivator- 1 beta in the pathogenesis of fructose-induced insulin resistance. Cell Metab. 9:252-264.

19. Severson, K. 2009 March 20. Sugar is back on food labels, this time as a selling point. The New York Times. http://www.nytimes.com/2009/03/21/ dining/21sugar.html 